Artigo

\title{
Diagnóstico Meteorológico dos Desastres Naturais Ocorridos nos Últimos 20 Anos na Cidade de Duque de Caxias
}

\author{
Priscila da Cunha Luz Barcellos ${ }^{1}$, Fabricio Polifke da Silva ${ }^{2}$, Fernanda Santa Bárbara Vissirini ${ }^{3}$, \\ Camila de Assis Magalhães ${ }^{3,4}$, Juliana Marques Terra ${ }^{4}$, Marcos Rezende Ferreira Dutra ${ }^{4}$, \\ Igor Cunha França do Amaral ${ }^{4}$ \\ ${ }^{1}$ Programa de Pós-Graduação em Engenharia Civil, Escola de Engenharia, \\ Universidade Federal Fluminense, Niterói, RJ, Brasil. \\ ${ }^{2}$ Programa de Engenharia Civil, Instituto Alberto Luiz Coimbra \\ de Pós-Graduação e Pesquisa de Engenharia, Rio de Janeiro, RJ, Brasil. \\ ${ }^{3}$ Secretaria Municipal de Defesa Civil e Políticas de Segurança de Duque de Caxias, \\ Prefeitura de Duque de Caxias, Duque de Caxias, RJ, Brasil. \\ ${ }^{4}$ Departamento de Meteorologia, Instituto de Geociências, \\ Universidade Federal do Rio de Janeiro, Rio de Janeiro, RJ, Brasil
}

Recebido: 01/12/2015 - Aceito: 08/03/2016

\begin{abstract}
Resumo
Este trabalho buscou auxiliar o planejamento de ações, medidas preventivas e mitigadoras, a desastres naturais para a Defesa Civil da cidade de Duque de Caxias, e utilizou a Classificação e Codificação Brasileira de Desastres (COBRADE) para identificação de 35 desastres naturais ocorridos entre os anos de 1996 a 2015, onde 74\% desses eventos ocorreram entre os meses de novembro e fevereiro, sendo $20 \%$ ocorridos no ano de 2009 . Para diagnosticar os sistemas meteorológicos, foram utilizados o Boletim de Climanálise do Centro de Previsão do Tempo de Estudos Climáticos - CPTEC, onde 57\% dos desastres foram associados à Zona de Convergência do Atlântico Sul (ZCAS), 29\% a passagem de frentes frias e $14 \%$ a áreas de instabilidade local. Objetivando subsidiar a confecção dos protocolos de acionamento de sirenes, foram analisadas a espacialização da precipitação ocorrida em 116 pluviômetros. Os resultados mostram que, para eventos de ZCAS, a faixa de maior frequência de acumulados diários de chuva variou entre $10 \mathrm{e}$ $50 \mathrm{~mm}$ nas $24 \mathrm{~h}$ antecedentes, e entre 60 e $110 \mathrm{~mm}$ no dia do desastre. Para frentes frias, os acumulados variaram entre 40 e $110 \mathrm{~mm}$, e para áreas de instabilidade entre 30 e $95 \mathrm{~mm}$.
\end{abstract}

Palavras-chave: desastres naturais, defesa civil, ZCAS.

\section{Weather Diagnosis of Natural Disasters Occurred in the Last 20 Years in Duque de Caxias City}

\begin{abstract}
This study aimed to assist the planning of actions, preventive and mitigating measures, the natural disasters for civil protection in the Duque de Caxias city, and used the Classification and Coding Brazilian Disaster (COBRADE) for identification of 35 natural disasters between the years from 1996 to 2015, where $74 \%$ of these events occurred between the months of November and February, $20 \%$ occurred in 2009. To diagnose the weather systems we used the Bulletin of Climate Studies of Weather Center - CPTEC, where $57 \%$ of disasters were associated with the South Atlantic Convergence Zone (SACZ), 29\% the passage of cold fronts and 14\% to local instability areas. The aim of assisting the preparation of sirens drive protocols, we analyzed the spatial distribution of rainfall occurred in 116 rain gauges. The results show that for SACZ event, the higher frequency range of daily rain accumulated ranged between 10 and $50 \mathrm{~mm}$ on the last $24 \mathrm{~h}$, and between 60 and $110 \mathrm{~mm}$ on the day of disaster. For cold fronts, retained varied between 40 and $110 \mathrm{~mm}$, and to areas of instability between 30 and $95 \mathrm{~mm}$.
\end{abstract}

Keywords: natural disasters, civil defense, SACZ. 


\section{Introdução}

Em todo o globo tem sido observado um aumento da ocorrência de desastres naturais e os prejuízos econômicos associados a eles. De acordo com a base de dados do Centro de Pesquisa em Epidemiologia de Desastres (EM-DAT, 2012), o aumento do número de desastres passou de 50 registros por ano na década de 60, chegando a 500 em 2005, e os prejuízos estimados aumentaram de 6 bilhões de dólares em 1965 para 300 bilhões em 2011.

Em 2015, o Relatório de Avaliação Global de 2015 (GAR, 2015) apontou que o investimento anual global de US\$ 6 bilhões em estratégias apropriadas para gestão de risco de desastres, geraria benefícios totais em termos de redução de risco de US\$ 360 bilhões. Baseado nestes dados, é indiscutível que os prejuízos econômicos decorrentes de desastres naturais são significativos, e os esforços empregados na prevenção, como a modernização de técnicas e do uso das informações do tempo e do meio ambiente, possibilitam a redução de perdas bilionárias na economia e perda de vidas. Investir na redução do risco de desastres é, portanto, um pré-condição para o desenvolvimento sustentável no contexto de mudanças climáticas.

De acordo com o Quinto Relatório de Avaliação do Painel Intergovernamental sobre Mudanças Climáticas (2014), os riscos relacionados aos eventos climáticos extremos, tais como ondas de calor, precipitação extrema e inundações costeiras, já são de moderado a alto, com um alto índice de confiança na incerteza das previsões. A intensificação da frequência dos desastres no mundo tem início na década de 1950, agravando-se nas décadas de 1980 e 2000. Estas observações indicam não só a elevação da frequência dos desastres, mas também a intensificação dos eventos extremos, e suas consequências para a sociedade (Carmo e Anazawa, 2014).

No Brasil, mais de $80 \%$ dos desastres naturais são desencadeados por fenômenos meteorológicos e os impactos sociais são função não somente dos eventos de origem atmosférica, mas também da ação humana (Pielke e Carbone, 2002), o que tem exigido permanente atenção dos órgãos de Defesa Civil nos últimos anos.

No Glossário da Defesa Civil Nacional, desastre é tratado como sendo "resultado de eventos adversos, naturais ou provocados pelo homem, sobre um ecossistema (vulnerável), causando danos humanos, materiais e/ou ambientais e consequentes prejuízos econômicos e sociais. A intensidade de um desastre depende da interação entre a magnitude do evento adverso e o grau de vulnerabilidade do sistema receptor afetado" (Castro, 1998). Desastres naturais, portanto, é caracterizado como uma função da ameaça, que no caso do Brasil é iniciada com um fenômeno extremo meteorológico, e da vulnerabilidade das comunidades, que, segundo Carmo e Anazawa (2014), encontra-se na origem de grande parte dos desastres, pois no Brasil há a inexistência de uma política habitacional capaz de dar conta das demandas por habitação digna e segura.
Eventos extremos sempre ocorreram, e a ocupação histórica de áreas de risco, aliada à falta de um sistema de alerta e de treinamento das populações para lidar com essas situações, oferece um ambiente propenso a tragédias (Projeto Megacidades, Vulnerabilidades e Mudanças Climáticas, 2011). O que era um processo de origem natural, como deslizamentos de encostas e inundações, se transforma num desastre e calamidade pública por consequência das alterações no meio físico da ação do homem.

Estudos como Kobiyama (2004), EM-DAT (2006) e Kobiyama et al. (2006) apontam, porém, que o número de perdas de vidas humanas ocasionadas por desastres naturais vem reduzindo, apesar do aumento da população, e isto se deve, em parte, a presença efetiva de ações preventivas em diversos setores, principalmente associadas às ações da Defesa Civil. Neste âmbito, verifica-se que o levantamento de desastres naturais se torna uma importante ferramenta de planejamento de ações, liberação de recursos e preparação de equipes para o enfrentamento de situações adversas, reduzindo os efeitos dos mesmos, e ainda, constituem-se uma base importante para estudos mais avançados, que visem análises locais detalhadas de vulnerabilidade e de risco, como também a análise da relação homem $/$ meio ambiente (Reckziegel, 2007). Desta forma, a formação de um banco de dados contextualizado, com as ocorrências de desastres classificadas de forma padronizada em todo o território nacional, permite o planejamento de medidas preventivas e mitigadoras, a partir do diagnóstico e conhecimento de eventos passados, e torna-se uma ferramenta importantíssima de base para as Defesas Civis Estaduais e Municipais, e também à população local.

Cientes da importância de estudar fenômenos meteorológicos como precursores de desastres naturais, autores pelo Brasil iniciaram esta investigação. Na região Sul do país, trabalhos como Nedel et al. (2012), realizaram um zoneamento dos desastres naturais ocorridos no estado do Rio Grande do Sul no período de 1989-2009 ocasionados pela ocorrência de granizo e vendavais. Utilizando os registros de ocorrência dos eventos obtidos junto à Coordenadoria Estadual de Defesa Civil, os autores conseguiram observar as regiões mais atingidas por eventos de granizo e vendavais, assim como, os meses mais favoráveis à ocorrência destes eventos, sendo tal informação corroborada como ferramenta útil para medidas de prevenção dos danos associados a estes eventos.

Na região Sudeste, Moura et al. (2013) realizaram uma classificação sinótica da sequência de campos de pressão ao nível médio do mar (PNMM) e de altura geopotencial em $500 \mathrm{hPa}$, associados à ocorrência de precipitação intensa na cidade do Rio de Janeiro - RJ, e mostraram que existem três tipos de padrões sinóticos que causaram eventos extremos de chuva na cidade entre 1997-2010, sendo o padrão dominante com a passagem de frentes frias com trajetória predominantemente zonal. Dereczynski et al. (2009) analisaram 160 eventos de chuvas intensas também 
para a cidade do Rio de Janeiro, constatando que, em $77 \%$ dos casos havia a atuação de sistemas frontais, 13\% dos casos estavam associados à Zona de Convergência do Atlântico Sul, 8\% à sistemas convectivos de mesoescala predominantes no verão e chuvas intensas geradas por efeito de circulação marítima ocorreram em apenas $2 \%$ dos casos.

Assim, este estudo buscou contribuir para uma compilação dos dados relacionados a desastres naturais na cidade de Duque de Caxias nos últimos 20 anos, correlacionando informações de vulnerabilidades nas comunidades com os fenômenos meteorológicos, enfatizando a análise temporal e espacial das chuvas extremas durante os desastres, com isso, auxiliando e aprimorando ações da Defesa Civil Municipal e medidas de mitigação para cidade.

\section{Material e Métodos}

\subsection{Caracterização da cidade de Duque de Caxias}

Com uma população estimada em 882.729 habitantes em 2015, podendo chegar a um milhão até 2020 (IBGE, 2015), o município de Duque de Caxias é o terceiro mais populoso do Estado do Rio de Janeiro, integrando a região Metropolitana do Rio de Janeiro, situado na Baixada Fluminense. A cidade apresenta características de baixada e tem reflexos da grande quantidade de áreas susceptíveis a ocorrências de desastres naturais, tanto nas áreas de topografia mais plana, com alto risco a cheias; quanto nas áreas montanhosas, com declividades acentuadas e índice de escorregamento elevado.

O município é dividido em quatro distritos: Duque de Caxias (centro), Campos Elíseos, Imbariê e Xerém; onde a maior concentração da população se apresenta principalmente nos dois primeiros distritos. A região está inserida no bioma Mata Atlântica, que se estendia desde as nascentes dos rios até a beira da Baía de Guanabara. Hoje, essa vegetação está praticamente limitada a uma parte do distrito de Xerém, com florestas altas e densas, ainda que perturbadas em alguns pontos. Para os demais distritos, a mata se limita a áreas de vegetação secundária e várzeas arbustivas nas planícies temporariamente encharcadas.

Com o marcante desenvolvimento industrial no município, juntamente com o entorno da Região Metropolitana do Rio de Janeiro, a cidade atraiu um grande contingente de imigrantes, que resultou na ocupação inadequada de áreas de encostas e de planícies fluviais. $\mathrm{O}$ processo de urbanização pode ser visto em lotes e quadras bem definidos na região central e de núcleos desordenados no restante do município, principalmente em encostas e nas planícies fluviais, predominando o padrão de moradias de média e baixa renda de autoconstrução (REGEA, 2013). Estas áreas apresentam hoje, alto risco a escorregamentos e inundações, pelo somatório dos fatores naturais e dos fatores de ocupação urbana desordenada.

\subsection{Classificação dos desastres naturais}

Primeiramente, foi realizada ampla pesquisa para a cidade de Duque de Caxias, entre os anos de 1996 a 2015 , na mídia impressa e digital disponível, com o intuito de buscar datas de eventos meteorológicos que se caracterizaram como desastres naturais. A partir destas datas, tomando por base a classificação de Desastre Naturais da Codificação Brasileira de Desastres (COBRADE, 2012) e dos critérios estabelecidos mundialmente pelo Centre for Research on the Epidemiology of Disasters - Cred (2013), para que um evento de chuva intensa com consequências para a população na cidade de Duque de Caxias fosse considerado desastre natural, foi considerado neste artigo que, pelo menos um dos seguintes critérios descritos foram atingidos: ocorrência de desabrigados e/ou desalojados, mortes, eventos de deslizamentos de terra ou transbordamento de rios (mesmo sem vítimas), declaração de situação de emergência, ou declaração de situação de calamidade pública.

\subsection{Dados utilizados no diagnóstico meteorológico}

Para a classificação e o diagnóstico meteorológico dos desastres naturais, foram utilizadas as informações do Boletim de Climanálise do Centro de Previsão do Tempo de Estudos Climáticos - CPTEC. Desta forma, o precursor meteorológico foi classificado como: frente fria, Zona de Convergência do Atlântico Sul (ZCAS) ou áreas de instabilidade, para cada um dos desastres relacionados anteriormente.

\subsection{Dados de precipitação}

Para subsidiar a confecção dos protocolos de acionamento de sirenes na cidade de Duque de Caxias, torna-se essencial analisar a quantidade de precipitação ocorrida em cada desastre. Para tal, buscou-se dados pluviométricos na região nos últimos 20 anos que pudessem ser representativos para as análises.

Levando em consideração a problemática da escassez de dados de chuva entre os anos de 1996 e 2007, bem como, as modificações geográficas e sociais ocorridas com frequência ao longo dos últimos anos na cidade, não foram analisados, visando a confecção dos protocolos de sirenes, os desastres ocorridos entre 1996 e 2007.

Para a realização da distribuição espaço-temporal da precipitação durante os desastres ocorridos entre 2008 e 2015, foi utilizado o software de interpolação de dados Surfer, com os dados de 29 estações pluviométricas do Instituto Estadual do Ambiente - INEA; 53 estações pluviométricas do Centro de Monitoramento e Alerta de Desastres Naturais- CEMADEN e 34 estações pluviométricas do Sistema Alerta Rio (Fig. 1).

Para cada desastre, foram confeccionados mapas de distribuição espacial com os acumulados em $24 \mathrm{~h}$ para o dia do desastre e para as $24 \mathrm{~h}$ antecedentes do evento (entende-se por acumulado em 24 h o somatório da chuva ocorrida 


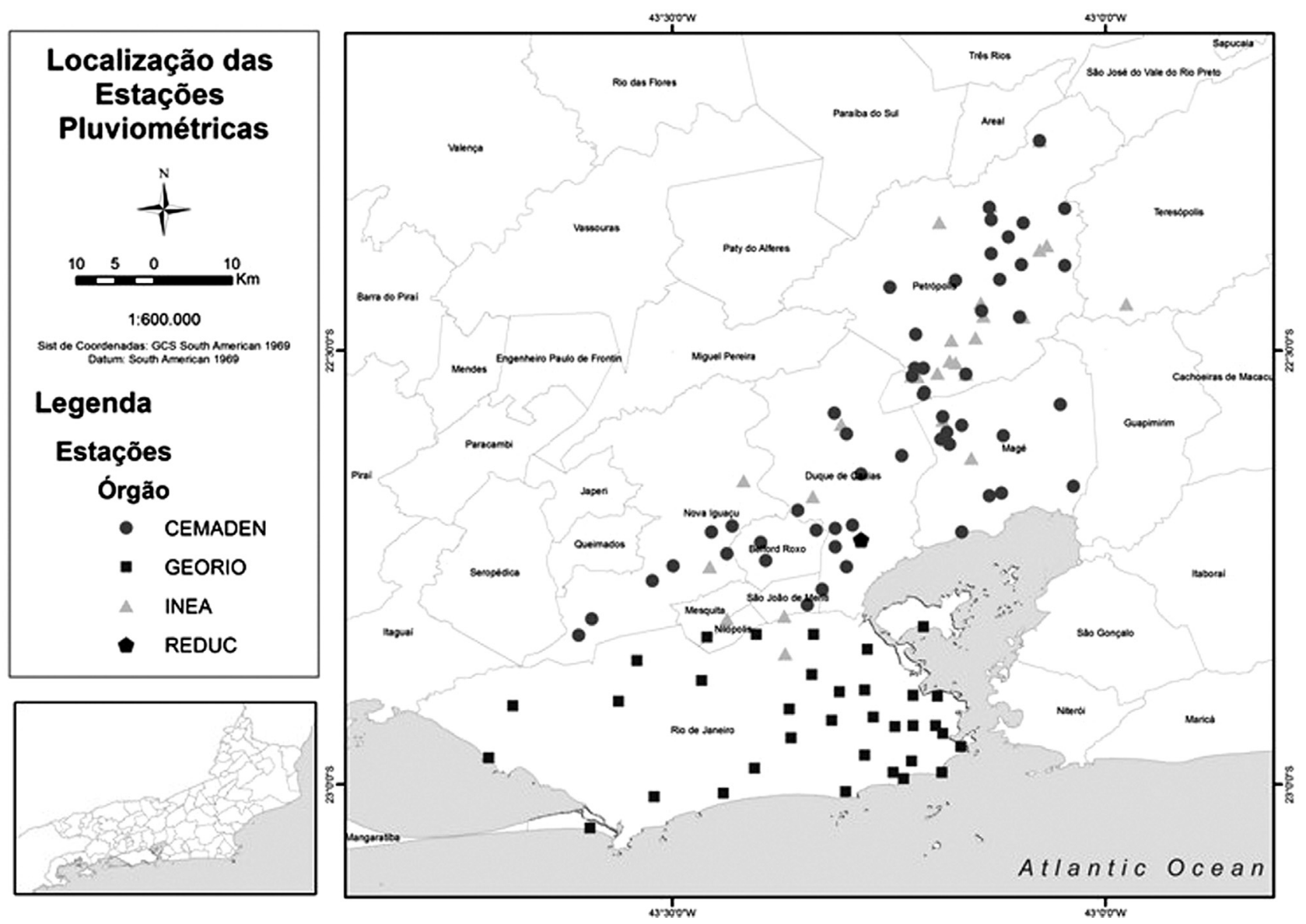

Figura 1 - Localização das estações pluviométricas do INEA, CEMADEN, GEORIO e REDUC que foram utilizadas para confeccionar a distribuição espacial da precipitação sobre a cidade de Duque de Caxias em cada evento mapeado.

entre 00:00 h e 23:59 h do dia analisado). Após a realização das interpolações pluviométricas, foram confeccionadas tabelas para a análise da frequência dos acumulados de chuva em 24 h, correlacionando cada evento com o diagnóstico meteorológico em questão.

\section{Resultados e Discussão}

\subsection{Classificação e distribuição dos desastres naturais}

Após a ampla pesquisa, foram classificados 35 eventos de desastres naturais ocorridos entre 1996 e 2015 na cidade de Duque de Caxias, conforme Tabela 1. Cabe ressaltar que nem todos os desastres naturais relacionados neste artigo tiveram um número significativo de pessoas afetadas, e que, com o objetivo de pesquisar o maior número de eventos meteorológicos severos na cidade, a classificação dos desastres naturais leva em consideração eventos de ocorrência de deslizamentos de terra e inundações dos rios, que ocasionaram transtornos a cidade, mesmo quando não houve perdas de vida, desabrigados ou desalojados.

Na maior parte do ano, o tempo no Estado do Rio de Janeiro permanece sob a influência do Anticiclone Subtropical do Atlântico Sul (ASAS) (FIDERJ, 1978). Esta situação de estabilidade é interrompida pela chegada de sistemas transientes que promovem o aumento da nebulosidade, ocorrência de precipitação e intensificação dos ventos. Nobre et al. (1998) destacam que a trajetória dos sistemas frontais sobre a América do Sul segue dois padrões bastante distintos ao longo do ano, sendo mais lenta no verão, caracterizada por maior atividade convectiva devido às complexas interações entre os sistemas transientes de escala sinótica e os sistemas quase estacionários como a Alta da Bolívia e a ZCAS, e com deslocamento mais rápido no inverno, onde os sistemas frontais deslocam-se em trajetórias mais zonais e não conseguem penetrar no interior do continente. Analisando mensalmente a ocorrência de desastres na cidade de Duque de Caxias, conforme o Gráfico 1, destaca-se que $74 \%$ desses eventos foram configurados entres os meses de novembro e fevereiro, caracterizando bem o período de verão chuvoso na região. Entre os meses de maio e setembro, caracterizados pelo regime de inverno, não houve registro de desastres naturais, o que corrobora com o período de seca das chuvas sobre o Estado do Rio de Janeiro.

Na distribuição anual (Gráfico 2), os anos de 2008 e 2009 apresentaram o maior número de recorrência de de- 
Tabela 1 - Diagnóstico meteorológico dos desastres ocorridos entre 1996 e 2015 na cidade de Duque de Caxias.

\begin{tabular}{|c|c|c|c|c|c|}
\hline Data do Evento & Análise Sinótica & Tipo de Desastre & Desabrigados & Desalojados & Mortes \\
\hline $19-23 / 11 / 1996$ & Frente fria & Deslizamento & 0 & 0 & 2 \\
\hline $18 / 12 / 1997$ & Frente fria & Inundação & 350 & 100 & 0 \\
\hline $07-09 / 01 / 1998$ & Frente fria & Deslizamento & 160 & 1000 & 6 \\
\hline 11/02/1998 & ZCAS & Deslizamento/Inundação & 1000 & 0 & 4 \\
\hline $09-10 / 12 / 1999$ & ZCAS & Inundação & 30 & 0 & 0 \\
\hline $23-24 / 12 / 2001$ & ZCAS & Deslizamento/Inundação & 0 & 1424 & 6 \\
\hline $02-03 / 02 / 2002$ & ZCAS & Inundação & 1600 & 0 & 1 \\
\hline $27-30 / 01 / 2003$ & ZCAS & Inundação & 0 & 0 & 0 \\
\hline $28-29 / 11 / 2003$ & Frente fria & Deslizamento/Inundação & 0 & 2000 & 7 \\
\hline $15-16 / 01 / 2004$ & ZCAS & Deslizamento & 269 & 1268 & 3 \\
\hline $27 / 01 / 2006$ & ZCAS & Deslizamento/Inundação & 0 & 0 & 3 \\
\hline 07/10/2006 & Frente fria & Deslizamento & 0 & 50 & 3 \\
\hline $24-25 / 10 / 2007$ & ZCAS & Deslizamento/Inundação & 0 & 1600 & 1 \\
\hline $12 / 12 / 2007$ & ZCAS & Deslizamento/Inundação & 0 & 0 & 0 \\
\hline 03/02/2008 & ZCAS & Deslizamento/Inundação & 0 & 0 & 0 \\
\hline $15 / 03 / 2008$ & ZCAS & Deslizamento & 0 & 0 & 0 \\
\hline 02/10/2008 & Áreas de instabilidade & Deslizamento & 0 & 0 & 0 \\
\hline $14 / 11 / 2008$ & ZCAS & Deslizamento & 0 & 0 & 0 \\
\hline $21 / 01 / 2009$ & ZCAS & Deslizamento & 0 & 0 & 0 \\
\hline $12 / 02 / 2009$ & Áreas de instabilidade & Inundação & 0 & 0 & 0 \\
\hline $11 / 11 / 2009$ & ZCAS & Deslizamento/Inundação & 0 & 0 & 0 \\
\hline $15 / 11 / 2009$ & Áreas de instabilidade & Deslizamento/Inundação & 205 & 3039 & 3 \\
\hline 04/12/2009 & ZCAS & Deslizamento/Inundação & 55 & 100 & 3 \\
\hline $28 / 12 / 2009$ & Áreas de instabilidade & Inundação & 0 & 0 & 0 \\
\hline $30-31 / 12 / 2009$ & Frente fria & Deslizamento/Inundação & 865 & 1600 & 1 \\
\hline $15 / 01 / 2010$ & ZCAS & Deslizamento/Inundação & 0 & 0 & 0 \\
\hline 06/04/2010 & Frente fria & Deslizamento/Inundação & 187 & 23 & 0 \\
\hline 05/04/2011 & ZCAS & Inundação & 0 & 0 & 0 \\
\hline 24/04/2011 & Frente fria & Inundação & 0 & 0 & 0 \\
\hline 02/01/2012 & ZCAS & Deslizamento/Inundação & 44 & 0 & 0 \\
\hline 03/01/2013 & Frente fria & Deslizamento/Inundação & 0 & 0 & 1 \\
\hline $18 / 03 / 2013$ & ZCAS & Deslizamento/Inundação & 149 & 347 & 33 \\
\hline $11 / 12 / 2013$ & ZCAS & Inundação & 0 & 7000 & 2 \\
\hline $28 / 02 / 2015$ & Frente fria & Deslizamento/Inundação & 0 & 0 & 0 \\
\hline $22 / 03 / 2015$ & Áreas de instabilidade & Deslizamento/Inundação & 0 & 0 & 0 \\
\hline
\end{tabular}

sastres naturais na cidade, totalizando $31,4 \%$ das ocorrências, seguidos pelo ano de 2013, com um total 8,6\%.

\subsection{Diagnóstico Meteorológico dos desastres naturais}

Relacionando o perigo potencial dos sistemas meteorológicos com os 35 desastres naturais ocorridos, identificou-se que $57 \%$ deles foram associados a formação da Zona de Convergência do Atlântico Sul (ZCAS), 29\% a passagem de frentes frias e $14 \%$ a áreas de instabilidade local (Fig. 2).
Destacando que o fenômeno ZCAS foi o que mais originou desastres naturais na cidade de Duque de Caxias, ressalta-se também que este fenômeno, segundo Kousky (1988), é mais comum entre os meses de novembro e março, caracterizado por uma banda de nebulosidade convectiva, que se estende em geral desde a Amazônia até o Oceano Atlântico; o que concorda com os resultados de recorrências mensais citados na análise acima.

O sistema ZCAS, que é típico dos meses de verão, mantem a convecção tropical mais acentuada (Kodama, 1992) e contribui para a geração e manutenção dos altos 
Distribuição mensal dos desastres naturais ocorridos entre os anos de 1996 e 2015 na cidade de Duque de Caxias

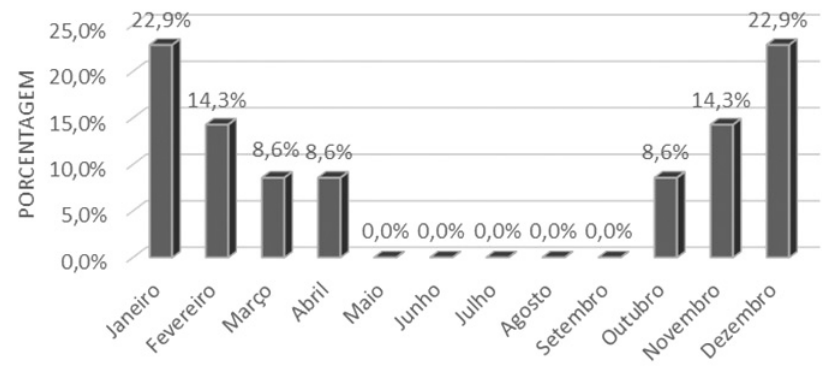

Gráfico 1 - Distribuição Mensal dos 35 desastres naturais ocorridos entre os anos de 1996 e 2015 na cidade de Duque de Caxias.

Distribuição Anual dos desastres naturais ocorridos entre os anos de 1996 e 2015 na cidade de Duque de Caxias

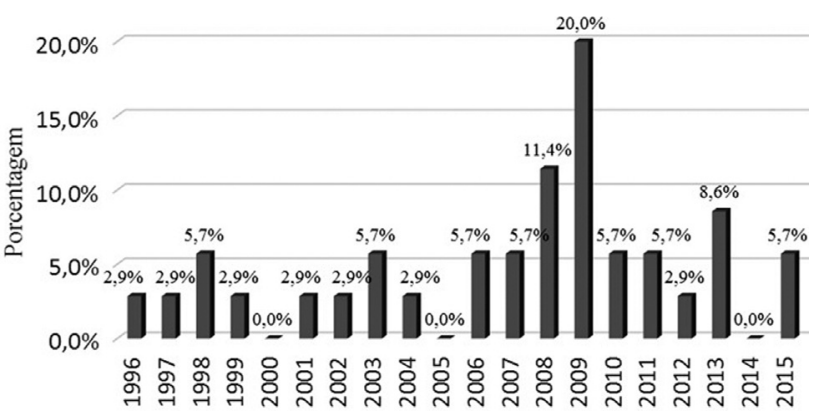

Gráfico 2 - Distribuição Anual dos 35 desastres naturais ocorridos entre os anos de 1996 e 2015 na cidade de Duque de Caxias.

\section{Diagnóstico Meteorológico dos Desastres Naturais}

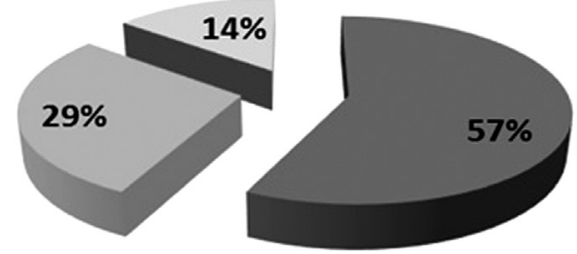

\section{$\square$ ZCAS $\square$ FRENTE FRIA $\square$ ÁREAS DE INSTABILIDADE}

Figura 2 - Diagnóstico meteorológico dos desastres naturais ocorridos entre os anos de 1996 e 2015 na cidade de Duque de Caxias.

acumulados de chuva por consecutivos dias, e que, somando-se aos problemas sociais e de infraestrutura das regiões vulneráveis a cheias e deslizamentos de terra na cidade de Duque de Caxias, se tornou o fenômeno mais contribuinte para a formação de desastres naturais na região.

\subsection{Desastre Natural em Duque de Caxias: Evento 03/01/2013}

No distrito de Xerém, em Duque de Caxias, na noite do dia 02 e madrugada do dia 03 de janeiro de 2013, a chegada de uma frente fria (Figs. 3a e 3b), intensificado pela convergência de umidade entre a faixa leste de SP e no sul do RJ, provocou chuva intensa em áreas da Região Serrana do e Baixada Fluminense. De acordo com a distribuição espacial da precipitação ocorrida durante o evento, o distrito de Xerém, em Duque de Caxias, foi o mais atingido na cidade, atingindo acumulados de precipitação maiores que $200 \mathrm{~mm}$ em apenas $8 \mathrm{~h}$ (Figs. 4a e 4b). Este evento de chuva intensa e extrema ocasionou uma rápida enxurrada nos rios João Pinto e Capivari, que desencadeou uma grande avalanche de terra, árvores, pedras e casas no bairro (Figs. 5a, 5b, 5c e 5d).

Esse desastre chamou a atenção de todo o Brasil, e se tornou um dos exemplos onde um evento natural de inundação se tornou em um desastre natural com grandes proporções, afetando centenas de pessoas, com perda de vida humana e deixando um rastro de destruição.

\subsection{Subsídio para o acionamento das sirenes: análise dos acumulados de chuva}

$\mathrm{O}$ acionamento de sirenes sonoras dentro das comunidades vulneráveis a desastres naturais é um trabalho que ganha destaque dentro da comunidade científica, pois possibilita que o monitoramento meteorológico seja utilizado para salvar vidas e propriedades. A importância deste trabalho, na gestão de risco de desastres e desocupação das comunidades vulneráveis, se dá, principalmente, devido a necessidade de maior confiança nos alertas por parte da população e, esses, precisarem ser os mais precisos possíveis.

Os protocolos de acionamento de sirenes são documentos que integram diversas técnicas, como por exemplo o mapeamento de risco das comunidades correlacionado com a intensidade e o acumulado de precipitação. Para sua confecção, são necessários longos históricos de dados de chuva e dados de vulnerabilidade sociais, a fim de se encontrar um valor médio em que há alta probabilidade do desastre ocorrer a partir de uma determinada intensidade e acumulado de chuva, na referida região de estudo.

Objetivando subsidiar a confecção dos protocolos de acionamento de sirenes na cidade de Duque de Caxias, foram interpolados dados de chuva de 116 pluviômetros (citados no item 2.4) para representar a distribuição espaço-temporal da precipitação no município de Duque de Caxias durante os eventos de desastres naturais entre os anos de 2008 e 2015.

Para os desastres originados por frentes frias, os resultados mostraram que os acumulados de precipitação em $24 \mathrm{~h}$ variaram entre 40 e $110 \mathrm{~mm}$ (Fig. 6), e para os desastres originados por áreas de instabilidade, os acumulados variaram entre 30 e $95 \mathrm{~mm}$ em 24 h (Fig. 7). 
(a)

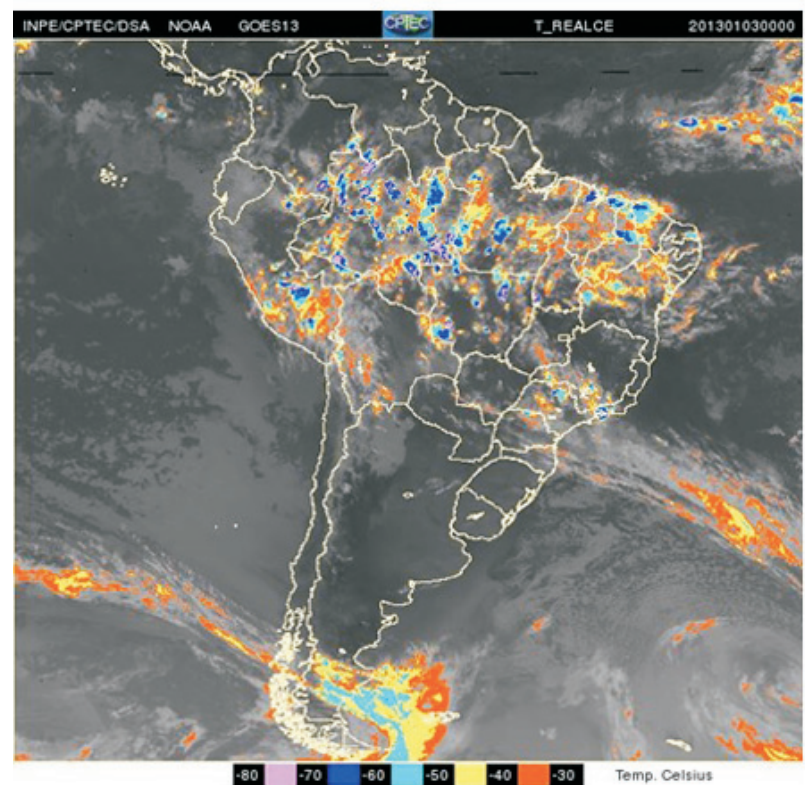

(b)

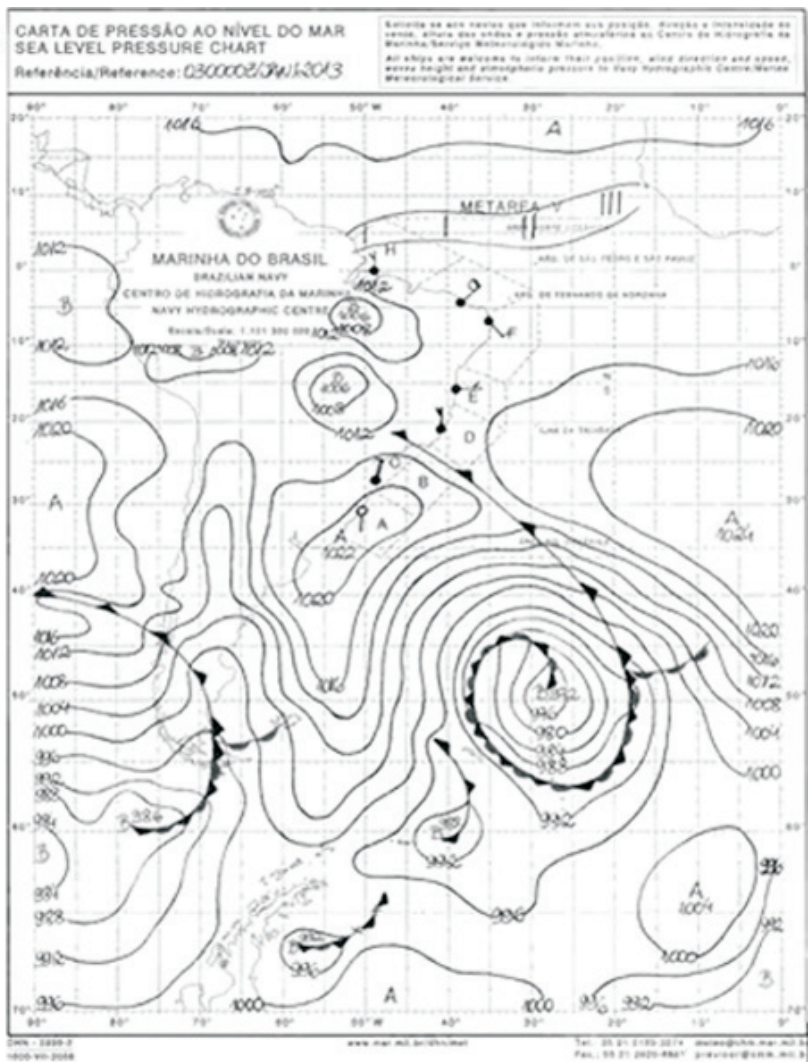

Figura 3 - Imagem realçada do satélite GOES (a) e carta sinótica da Marinha do Brasil (b) do dia 03 de janeiro de 2013 às 0:0 h.
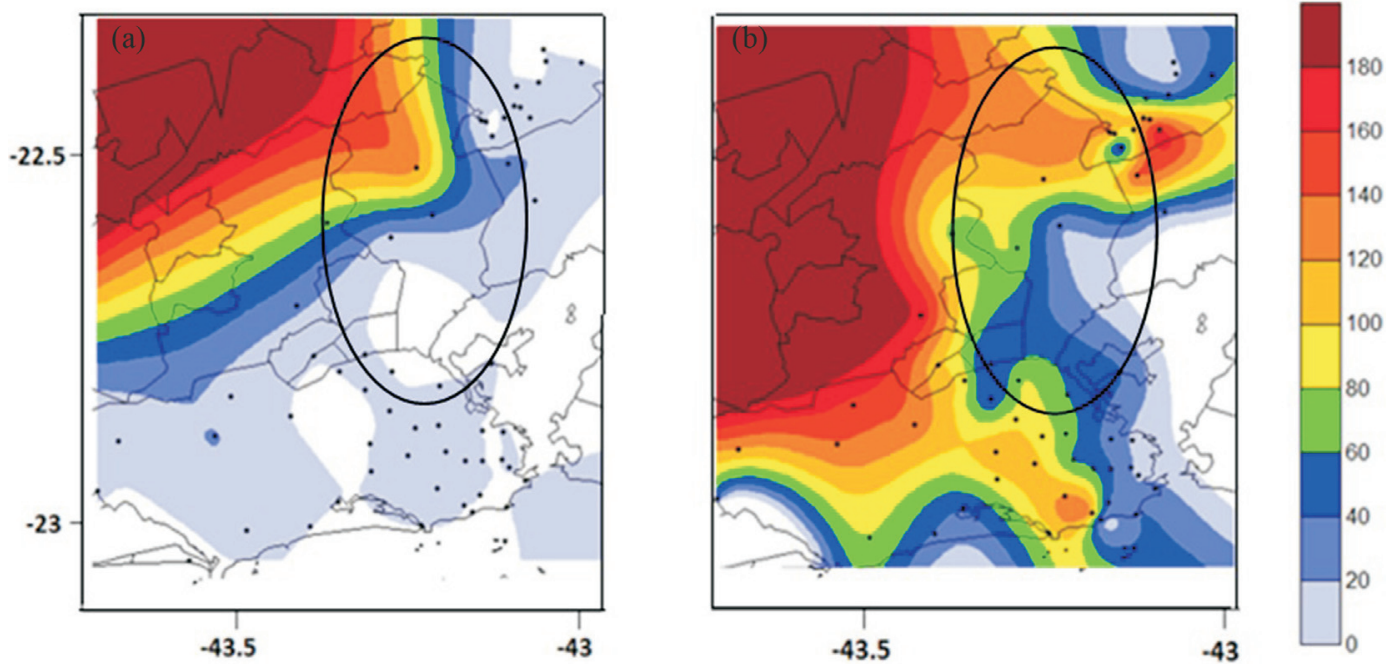

Figura 4 - Distribuição espacial da precipitação acumulada em 24 h nos dias 02 de janeiro (a) e 03 de janeiro (b) de 2013 na Duque de Caxias.

É curioso destacar que, mesmo acumulados teoricamente baixos como 40 e $30 \mathrm{~mm}$ em $24 \mathrm{~h}$, que foram os mínimos registrados pelos casos de frente fria e áreas de instabilidade, respectivamente, já foram suficientes para caracterizarem desastres naturais na cidade de Duque de Caxias. Estes resultados ressaltam a problemática da cidade não só sob o ponto de vista natural, mas também de infraestrutura e social, pois alguns bairros da cidade e algumas comunidades, como por exemplo São Bento e Jardim Anhangá, sofrem com a má drenagem urbana associada a problemas de ocupação irregular, agravando as consequências associadas aos fenômenos meteorológicos que não são considerados como tão extremos, mas que ainda assim ocasionam desastres.

No caso dos desastres ocasionados pela ZCAS, como a recorrência de pancadas de chuva por vários dias conse- 

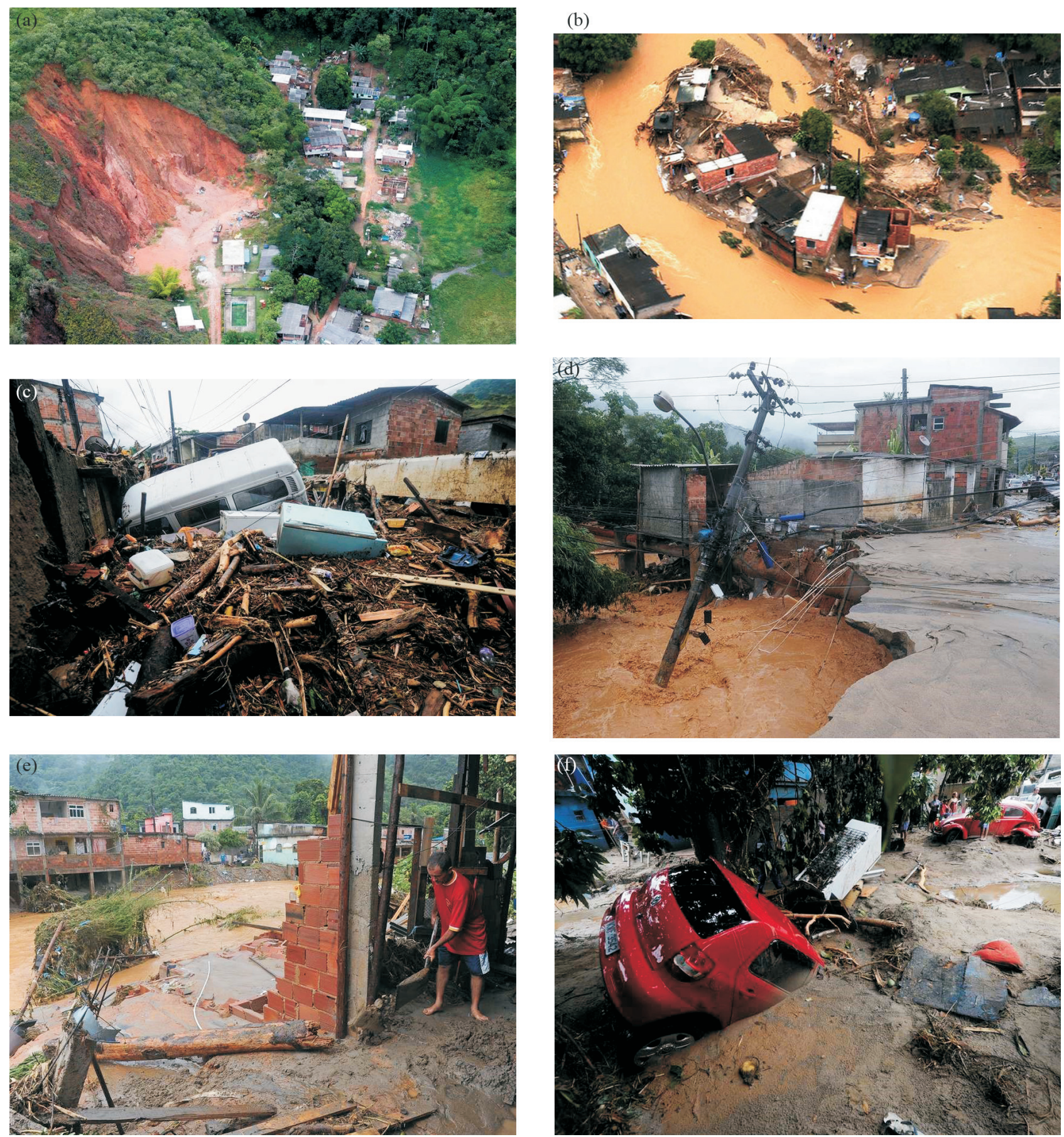

Figura 5 - Fotos do desastre natural ocorrido no bairro de Xerém, Duque de Caxias, no dia 03 de janeiro de 2013. (a) Foto de Marino Azevedo/Governo do RJ. (b) Foto da Revista Veja: imagem aérea da devastação nas margens do Rio Capivari, distrito de Xerém, município de Duque de Caxias. (c-f) Fotos de Daniel Ramalho/Terra.

cutivos é característica do fenômeno meteorológico, e este acumulado de chuva no solo e nos cursos hídricos é contribuinte e significativo para a ocorrência dos desastres, foram feitas análises sobre a faixa de frequência de acumulados diários de chuva no dia da ocorrência do desastre e no dia anterior.

Os resultados mostraram que os acumulados de precipitação variaram entre 10 e $50 \mathrm{~mm}$ nas $24 \mathrm{~h}$ que antece- deram o desastre, e entre 60 e $110 \mathrm{~mm}$ no dia do desastre para a cidade (Fig. 8). A partir dessas análises, o órgão de Defesa Civil já saberá de antemão que se já existirem acumulados de chuva dessa ordem (50 mm), e ainda são previstos acumulados maiores que $50 \mathrm{~mm}$ para as próximas horas, é alta a probabilidade de ocorrer um desastre novamente. 


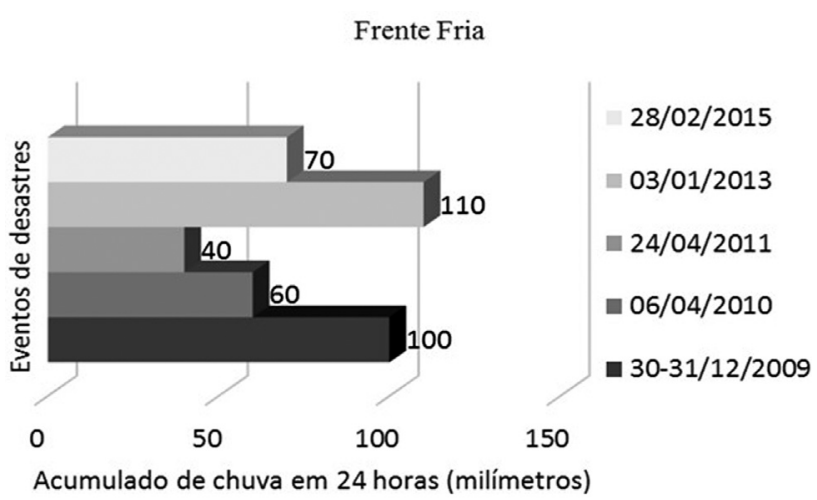

Figura 6 - Análise dos acumulados de chuva por evento para desastres originados por Frente Fria na cidade de Duque de Caxias.

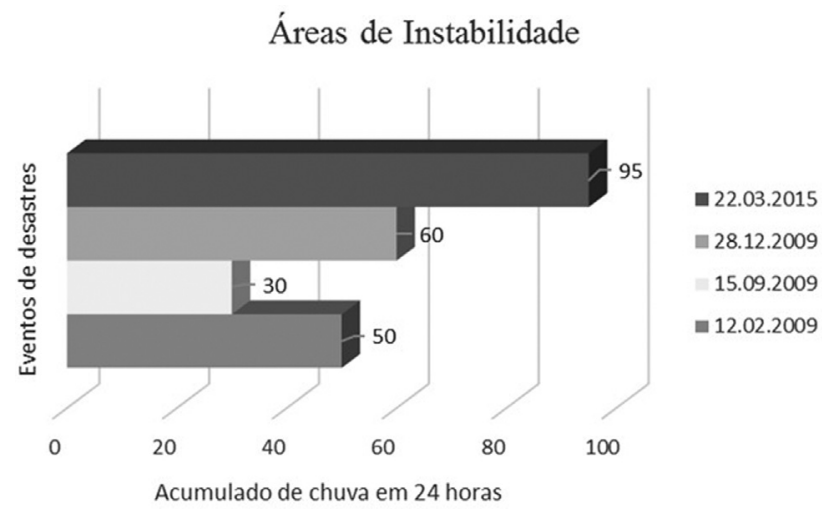

Figura 7 - Análise dos acumulados de chuva por evento para desastres originados por Áreas de Instabilidade na cidade de Duque de Caxias.

\section{Conclusões}

A análise de eventos meteorológicos extremos que ocasionam desastres naturais é de suma importância para auxiliar o planejamento de ações públicas, medidas preven- tivas e mitigadoras nas comunidades vulneráveis, e desta forma reduzir perdas de vidas e materiais.

Diante das mudanças climáticas e do apontamento da intensificação dos extremos sobre o Brasil, este trabalho buscou auxiliar a Defesa Civil da cidade de Duque de Caxias, pesquisando e identificando 35 desastres naturais ocorridos entre os anos de 1996 a 2015, e, inicialmente, relacionando-os com o precursor meteorológico.

Os resultados mostraram que $74 \%$ desses eventos ocorreram entre os meses de novembro e fevereiro, sendo $20 \%$ ocorridos no ano de 2009 , onde $57 \%$ dos desastres foram associados à Zona de Convergência do Atlântico Sul (ZCAS), 29\% a passagem de frentes frias e 14\% a áreas de instabilidade local.

Através das características típicas da Zona de Convergência do Atlântico Sul é importante destacar que a geração e manutenção dos altos acumulados de chuva por dias consecutivos, somado aos problemas sociais e de infraestrutura da cidade de Duque de Caxias, torna o fenômeno como o mais contribuinte para a formação de desastres naturais na região.

Posteriormente, o trabalho buscou subsidiar a confecção dos protocolos de acionamento de sirenes na cidade, e foram analisadas a espacialização da precipitação ocorrida com 116 pluviômetros em 21 desastres entre os anos de 2008 e 2015 . Os resultados mostraram que, para eventos de ZCAS, a faixa de maior frequência de acumulados diários de chuva variou entre 10 e $50 \mathrm{~mm}$ nas $24 \mathrm{~h}$ antecedentes, e entre 60 e $110 \mathrm{~mm}$ no dia do desastre. Para frentes frias, os acumulados variaram entre 40 e $110 \mathrm{~mm}$, e para áreas de instabilidade entre 30 e $95 \mathrm{~mm}$.

Com os resultados apresentados, esta pesquisa buscou produzir conhecimento científico acerca da evolução espaço-temporal dos desastres do tipo deslizamento e inundação, para a cidade de Duque de Caxias, concluindo-se que é possível relacionar os desastres naturais ocorridos na cidade com os eventos meteorológicos atuantes e assim,

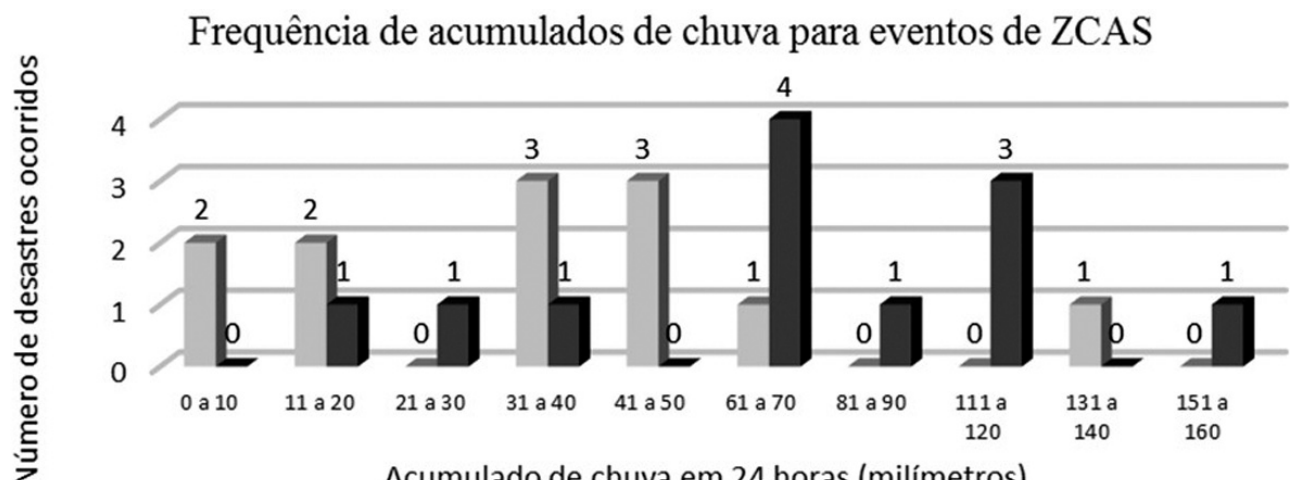

Acumulado de chuva em 24 horas (milímetros)

\section{$\square$ 24h anteriores $\square$ Dia do desastre}

Figura 8 - Frequência dos acumulados de chuva para os eventos de ZCAS na cidade de Duque de Caxias, 24 h anteriores e no dia da ocorrência do desastre natural. 
fomentar as informações sobre desastres naturais e contribuir para o processo de tomada de decisão dos gestores públicos e da sociedade civil.

Sugere-se como trabalhos futuros, análises e estudos mais aprofundados no assunto como a correlação dos anos com mais desastres e as influências do El Niño e La Niña e a investigação horária dos acumulados de chuva para cada desastre, buscando identificar efeitos mais localizados sobre o município e aumentar a capacidade de gerenciar riscos na cidade.

\section{Agradecimentos}

À Secretaria Municipal de Defesa Civil e Políticas de Segurança de Duque de Caxias, em especial ao Secretário Cel BM Marcello Silva da Costa, e à Prefeitura de Duque de Caxias representada pelo Excelentíssimo Prefeito Alexandre Cardoso, pelo incentivo e apoio irrestrito, bem como pela oportunidade para a realização e divulgação desse estudo.

\section{Referências}

CARMO, R.L.; ANAZAWA, T.M. Mortalidade por desastres no Brasil: o que mostram os dados. Ciência \& Saúde Coletiva 19(9):3669-3681, 2014.

CASTRO, A.L.C. Glossário de defesa civil: estudo de riscos e medicina de desastres. Brasília, MPO/ Departamento de Defesa Civil. 283 p. 1998.

CLASSIFICAÇÃO E CODIFICAÇÃO BRASILEIRA DE DESASTRES - COBRADE. 2012. Disponível em: http://www.mi.gov.br/defesa-civil/legislacoes. Acesso em: 15 mar. 2015.

CENTER FOR RESEARCH ON THE EPIDEMIOLOGY OF DISASTERS - CRED. Annual Disaster Statistical Review 2013 - The numbers and trends, 2013. Disponível em: http://www.cred.be/sites/default/files/ADSR_2013.pdf. Acesso em: 15 nov. 2015.

CENTRO DE MONITORAMENTO E ALERTA DE DESASTRES NATURAIS - CEMADEN. Série de dados dos pluviômetros. Disponível em: http://www.cemaden.gov.br/. Acesso em: 20 mar .2015.

CENTRO DE PREVISÃO E ESTUDOS DO CLIMA - CPTEC. Boletins de Climanálise. Disponível em: http://climanalise.cptec.inpe.br/ rclimanl/boletim/. Acesso em: 20 mai. 2015.

DERECZYNSKI, C.P.; OLIVEIRA, J.S.; MACHADO, C.O. Climatologia da precipitação no município do Rio de Janeiro. Revista Brasileira de Meteorologia, v.24, n.1, p. 24-38, 2009.

EMERGENCY EVENTS DATABASE - EM-DAT. Produce a list of disasters and associated losses. 2006. Disponível em: http://www.em-dat.net/links/disasterdbs.html. Acesso em: 01 nov .2015

EMERGENCY EVENTS DATABASE - EM-DAT. The OFDA/CRED International Disaster Database. Disponível em: http://www.em-dat.net/. Acesso em: 01 nov .2015.

FUNDAÇÃO GEOTÉCNICA DA CIDADE DO RIO DE JANEIRO - Sistema Alerta Rio. Série de dados dos pluviô- metros. Disponível em: http://alertario.rio.rj.gov.br/. Acesso em: 18 mar .2015.

FUNDAÇÃO INSTITUTO DE DESENVOLVIMENTO ECONÔMICO E SOCIAL DO RIO DE JANEIRO. Indicadores Climatológicos do Estado do Rio de Janeiro. Governadoria do Estado do Rio de Janeiro, Secretaria de Planejamento e Coordenação Geral (SECPLAN), Fundação Instituto de Desenvolvimento Econômico e Social do Rio de Janeiro (FIDERJ). Sistemas de Informação para o Planejamento Estadual (SIPE). Rio de janeiro, 1978. 156 p.

GLOBAL ASSESSMENT REPORT ON DISARTER RISK REDUCTION - GAR. 2015. Disponível em: http://reliefweb.int/sites/reliefweb.int/files/resources/GAR15_Pocket_EN.pdf Acesso em: 01 nov. 2015.

INSTITUTO BRASILEIRO DE GEOGRAFIA E ESTATÍSTICA - IBGE, 2015. Disponível em: http://cidades.ibge.gov.br/xtras/perfil.php?codmun = 330170. Acesso em: 05 nov. 2015.

INSTITUTO ESTADUAL DO AMBIENTE - INEA, Sistema de Alerta de Cheias do Estado do Rio de Janeiro. Série de dados dos pluviômetros. Disponível em: http://www.alertadecheias.com.br/. Acesso em: 20 mar. 2015.

INTERGOVERNMENTAL PANEL ON CLIMATE CHANGE IPCC. Quinto Relatório de Avaliação do Painel Intergovernamental sobre Mudanças Climáticas. 2014. Disponível em: http://www.ipcc.ch/index.htm. Acesso em: 20 mar. 2015.

KOBIYAMA, M.; CHECCHIA, T.; SILVA, R.V.; SCHRÖDER, P.H.; GRANDO, Â.; REGINATTO, G.M.P. Papel da comunidade e da universidade no gerenciamento de desastres naturais. In: Simpósio Brasileiro de Desastres Naturais, 2004, Florianópolis. Anais. Florianópolis: GEDN/UFSC, 2004. p. 834-846.

KOBIYAMA, M.; MENDONÇA, M.; MORENO, D.A.; MARCELINO, I.P.V.O.; MARCELINO, E.V.; et al. Prevenção de desastres naturais: conceitos básicos. Curitiba: Ed. Organic Trading, 2006. 109p.: il., tabs. Inclui bibliografia ISBN - 85-87755-03-X. Disponível em: http://www.disaster-info.net/lideres/portugues/curso-brasil08/documentos_e _artigos/Prevencao\%20desastres\%20naturais.pdf Acesso em: 08 nov. 2015.

KODAMA, Y. Large-Scale Common Features of Subtropical Precipitation Zones (the Baiu Frontal Zones, the SPCZ, and the SACZ) Part I: Characteristics of Subtropical Frontal Zones. Journal of Meteorological Society of Japan, v. 70, n.4, p. 813-835, 1992.

KOUSKY, V.E. Pentad Outgoing Longwave Radiation Climatology for the South American Sector. Revista Brasileira de Meteorologia, v. 3, p. 217-231, 1988.

MOURA, C.R.W.; ESCOBAR, G.C.J.; ANDRADE, K.M. Padrões de circulação em superfície e altitude associados a eventos de chuva intensa na região metropolitana do Rio de Janeiro. Revista Brasileira de Meteorologia, v.28, n.3, p. $267-280,2013$

NEDEL, A.; SAUSEN, T. M.; SAITO, S.M. Zoneamento dos desastres naturais ocorridos no estado do Rio Grande do Sul no período 1989-2009: Granizo e vendaval. Revista Brasileira de Meteorologia, v.27, n.2, p. 119 - 126, 2012.

NOBRE, C.A.; MATTOS, L.F.; DERECZYNSKI, C.P.; TARASOVA, T.A.; TROSNIKOV, I.V. Overview of atmo- 
spheric conditions during the Smoke, Clouds, and Radiation-Brazil (SCAR-B) field experiment. Journal of Geophysical Research, v. 103, n. D24, p. 31809-31820, 1998.

PIELKE JR., R.A.; CARBONE, R. "Weather impacts, forecasts and policy: an integrated perspective", Bulletin of the American Meteorological Society, v. 83, n.12,(Mar.), p. 393-402, 2002.

PORTAL ESTADÃo DE SÃo PAULO. Pesquisa de desastres na Baixada Fluminense. Disponível em: http://www.estadao.com.br/. Acesso em: 01 mai.2014.

PORTAL FOLHA DE SÃo PAULO. Pesquisa de desastres na Baixada Fluminense. Disponível em: http://www.folha.uol.com.br/. Acesso em: 01 mai.2014.

PORTAL G1. Pesquisa de desastres na Baixada Fluminense. Disponível em: http://g1.globo.com/index.html. Acesso em: 01 mai.2014.
PORTAL R7. Pesquisa de desastres na Baixada Fluminense. Disponível em: www.r7.com/. Acesso em: 01 mai.2014.

PROJETO MEGACIDADES, VULNERABILIDADES E MUDANÇAS CLIMÁTICAS. 2011. Disponível em: http://www.poli.ufrj.br/noticias/arquivos/completo.pdf. Acesso em: 03 nov. 2015.

RECKZIEGEL, B.W. Levantamento dos desastres desencadeados por eventos naturais adversos no Estado do Rio Grande do Sul no período de 1980 a 2005. Dissertação de Mestrado. PPGG. Santa Maria, Rio Grande do Sul, 2007. 261 p. Disponível em: http://w3.ufsm.br/ppggeo/files/dissertacoes_06-11/Bernade teReckziegel.pdf. Acesso em: 20 nov. 2015.

REGEA. Programa "Apoio à prevenção e erradicação de riscos em assentamentos precários" - Plano Municipal de Redução de Risco. Município de Duque de Caxias. Ministério das Cidades. 2013.

All the contents of this journal, except where otherwise noted, is licensed under a Creative Commons Attribution License CC-BY. 